\title{
Rotating Stall in Centrifugal Compressor Vaneless Diffuser: Experimental Analysis of Geometrical Parameters Influence on Phenomenon Evolution
}

\author{
Giovanni Ferrara and Lorenzo Ferrari \\ Department of Energy Engineering "S.Stecco," University of Florence, Florence, Italy \\ Leonardo Baldassarre \\ Centrifugal Compressor Division, GE—Nuovo Pignone, Florence, Italy
}

The rotating stall is a key problem for achieving a good working range of a centrifugal compressor and a detailed understanding of the phenomenon is very important to anticipate and avoid it. Many experimental tests have been planned by the authors to investigate the influence on stall behavior of different geometrical configurations. A stage with a backward channel upstream, a 2-D impeller with a vaneless diffuser and a constant cross-section volute downstream, constitute the basic configuration. Several diffuser types with different widths, pinch shapes, and diffusion ratios were tested. The stage was instrumented with many fast response dynamic pressure sensors so as to characterize inception and evolution of the rotating stall. This kind of analysis was carried out both in time and in frequency domains. The methodology used and the results on phenomenon evolution will be presented and discussed in this article.

Keywords Rotating stall, Centrifugal compressor, Vaneless diffuser, Dynamic pressure sensor

\section{INTRODUCTION}

Vaneless diffuser rotating stall generally occurs as a result of 3-D separation of boundary layer and consequent reverse

Received 26 February 2002; accepted 1 August 2002.

Our thanks to Prof. M. De Lucia and Prof. E. Carnevale for their encouragement during the research. Further thanks go to Stefano Vanghi and Moreno Soldani for test rig management and set-up.

Address correspondence to Giovanni Ferrara, Department of Energy Engineering “S.Stecco," University of Florence, Via S. Marta 3, 50139 Florence, Italy. E-mail: g.ferrara@ing.unifi.it flow. This kind of stall has been theoretically and experimentally studied by Jansen (1964), who was the first to show that diffuser rotating stall could be triggered by local inversion of the radial velocity component in the vaneless diffuser. He assumed the flow to be symmetric with respect to the diffuser depth. This assumption was not satisfactory since further studies (Senoo and Kinoshita, 1977; Senoo et al., 1977) show how, in most cases, local reverse flow occurs firstly in the hub-side wall in the diffuser inlet region, whereas it transfers from hub to shroud side downstream the inlet region. The boundary layer calculation was thus repeated by Senoo and Kinoshita (1977) with the following modifications: flow is no longer assumed to be symmetric with respect to the diffuser center, inlet conditions account for distortions of radial and tangential velocity over the diffuser width, and boundary layer profiles are modified in such a way that merging of the two boundary layers does not necessary lead to return flow. These calculations identify a critical velocity angle for which reverse flow starts in the diffuser (velocity field angle with respect to the radial direction in the boundary layer greater than $90^{\circ}$ ). On the basis of comparison with experimental data, Senoo and Kinoshita (1978) claim that the vaneless diffuser rotating stall will occur when the diffuser inlet flow angle is 0.88 times the previous identified critical angle. Some discrepancies between the predicted and measured critical angles are present. They were often linked to the passage between the impeller exit and the diffuser inlet. Senoo and Kinoshita's formulation does not consider the influence of these factors. This problem was solved experimentally by Nishida et al. (1988) and Kobayashi et al. (1990). The authors give a formula to evaluate the critical angle starting from Senoo's one and taking into account the diffuser geometries in terms of inlet shape and width.

In this context, a collaboration between General ElectricNuovo Pignone (GE-NP) and the Department of Energy Engineering "Sergio Stecco" (DE) was established: A wide ranging experimental campaign managed by University staff on a 
GE-NP test rig was planned in order to characterize the performance and phenomena of instability (stall, rotating stall, and surge) in the last high-pressure stages.

A 2-D impeller (diameter of $300 \mathrm{~mm}$ ) with free vortex diffuser and volute made up the model for the first part of the research. For reproducing the real stage inlet flow conditions a return channel upstream the impeller was also present. Several kinds of diffuser geometries were tested with different diffusion ratio, width, and pinch. The influence of these parameters on machine performance was analyzed. The model was also instrumented with several dynamic pressure sensors allowing an accurate analysis of stall phenomenon: inception, evolution, and characteristics. Dynamic sensors were placed following the considerations of Camp et al. (1998) so as to study stall evolution in each section and through the sections. In particular, a $90^{\circ}$ portion of the diffuser was widely instrumented to get the highest amount of information about stall evolving in it. In this article, the methodology utilized together with the results obtained with this analysis are proposed.

\section{TEST RIG FACILITIES}

The tests were performed in a closed loop test rig in which air flows at ambient temperature and pressure. The impeller was driven by a $370 \mathrm{~kW}$ variable-speed electric motor (max 3000 RPM) coupled with a gearbox. Details of the stage configurations tested will be given in the following pages. Downstream from the stage, the following elements were present (Figure 1):

1. The spot at which the test rig mass is reintegrated. This mass flow rate was measured by a calibrated diaphragm.

2. A calibrated diaphragm for the main mass flow rate measurement.

3. A water heat exchanger for maintaining the stage inlet temperature roughly constant.

4. A throttle valve to set operating points.

5. A plenum to eliminate stage inlet flow distortion.

Leakage coming from impeller back seal was collected (through a pipe), measured by a calibrated diaphragm, and discharged in ambient. This measurement was necessary to evaluate diffuser mass flow rate (equal to the difference between the main and the leakage mass flow rates). All the calibrated di-

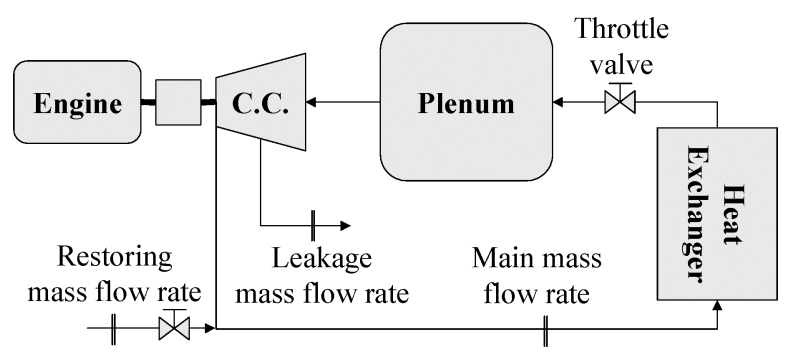

FIGURE 1

Test rig sketch. aphragms and measurement methodology were done according to ISO 5167 (1980).

\section{STAGE CHARACTERISTICS}

The model reproduces, on 1:1 geometrical scale, the last stage of an industrial centrifugal compressor for high-pressure applications (supplied pressure up to 600 bars).

A return channel, a 2-D impeller, a vaneless diffuser, and a constant cross-section volute constitute the basic conformation. The impeller is characterized by a small blade outlet width on impeller radius ratio: $b_{2} / R_{2}=0.0313$.

In Figure 2, a cross section of the stage with measurement sections is shown.

\section{TEST VARIABLES AND PROGRAM}

Reducing the diffuser width and modifying the pinch shape are common operations carried out to improve the diffuser stability. Tested diffuser widths were chosen in order to cover usual industrial machine requirements. Three diffuser widths were tested characterized by $\mathrm{b}_{20} / \mathrm{b}_{2}=0.30, \mathrm{~b}_{20} / \mathrm{b}_{2}=0.64$, and $\mathrm{b}_{20} / \mathrm{b}_{2}=0.38$ that was expected to have stall roughly in the middle of the working range.

Starting from the observation that, for narrow diffuser, stall inception occurs close to impeller discharge, it can be concluded that pinch shape could play a key role in diffuser stability. The more rapid the pinch (the area change with respect to the radial extent), the more stable the diffuser should be. According to this consideration a pinch $(r a)$ with a quarter of circumference as inlet profile was realized. With respect to the GE-NP standard geometry (pinch $n p$-almost linear), the $r a$ pinch obviously leads to higher pressure losses and consequently worse overall performance. For this reason a pinch

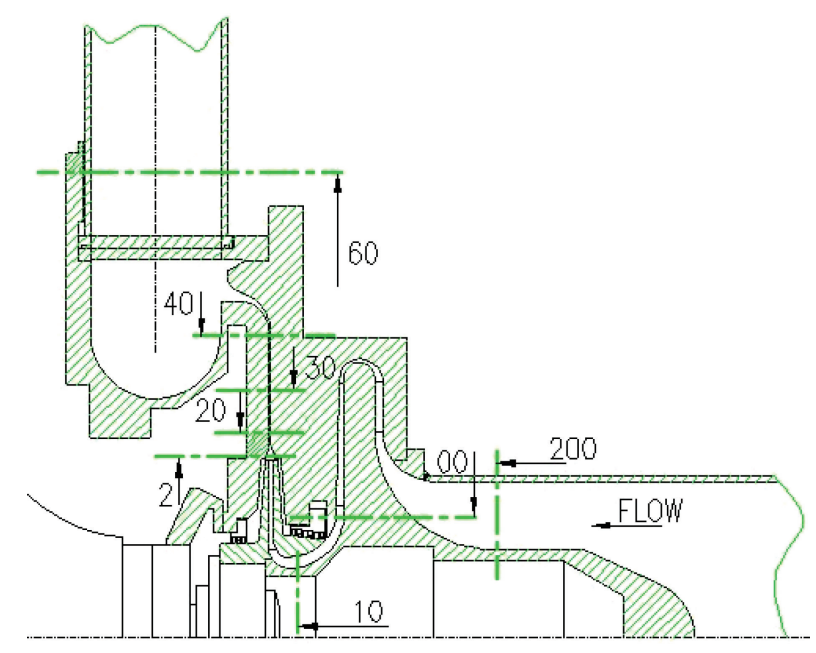

FIGURE 2

Meridian view of the model and measurement sections, upper side. 

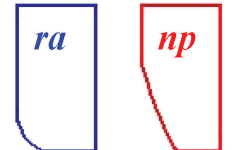

in

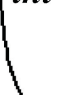

FIGURE 3

Pinch shapes $(r a, n p, i n t)$ and comparison.

(int) with an intermediate shape (an ellipse arc) between the two previous shapes was designed and tested. This pinch is supposed to show the best results in terms of diffuser stability and overall machine performance. Pinch shapes are reported in Figure 3.

In addition to the above-mentioned variables, the influence of diffusion ratio was also analyzed. From the industrial point of view, succeeding in reducing the overall machine dimension means reducing the machine cost, especially for high-pressure machines. For this reason, a configuration with reduced diffusion ratio ( $\mathrm{DR}=1.3$ ) could be interesting from an industrial point of view. The shorter diffuser should also have a positive impact on diffuser stability according to Abdelhamid (1983). Two diffusion ratios (DR) were tested: $\mathrm{DR}=1.3$ and $\mathrm{DR}=1.7$.

Two different volutes were used for the two DR. Different radii characterized them whereas the area and the shape of cross section were the same.

To ensure similarity between testing and design conditions, the peripheral Mach reference number $\mathrm{M}_{\mathrm{u}}=0.5$ was chosen. However, tests were performed at two different values for the peripheral Mach number. Since the test was done in ambient condition, the Reynolds number was not the same of high-pressure industrial machine. Regardless Senoo et al. (1977) have shown that tall behavior is Reynolds number independent for small $\mathrm{b}_{2} / \mathrm{R}_{2}$. All test variables are reported in Table 1 .

\section{MODEL INSTRUMENTATION}

Sections reported in Figure 2 were instrumented as reported in Table 2. The instrumentation was selected to achieve the following:

- performance evaluation of the whole stage and of each component

TABLE 1

Stage Characteristics and Test Variables

\begin{tabular}{lcll}
\hline Impeller radius & $\mathrm{R}_{2}$ & $150 \mathrm{~mm}$ & \\
$\begin{array}{l}\text { Outlet blade width on } \\
\quad \text { impeller radius }\end{array}$ & $\mathrm{b}_{2} / \mathrm{R}_{2}$ & 0.0313 & \\
Diffusion ratio & & & \\
Dim.less diffuser width & $\mathrm{DR}$ & $1.3-1.7$ & \\
& $\mathrm{D}$ & $\mathrm{A}$ & 0.30 \\
& & $\mathrm{~B}$ & 0.38 \\
Pinch shape type & & $\mathrm{C}$ & 0.64 \\
Peripheral Mach Number & $\mathrm{P}$ & $n p-$ int-ra & \\
\hline
\end{tabular}
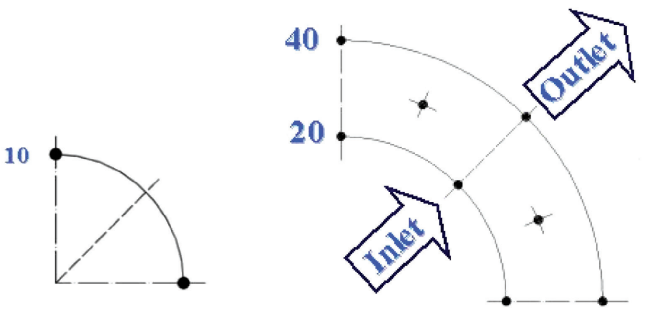

FIGURE 4

Detail of diffuser instrumentation DR $=1.3$.

- clear detection of component where rotating stall first starts

- evaluation of the flow critical angle at diffuser inlet

Due to the narrow diffuser widths, it was not possible to use three-hole probes for the flow angle estimation. Thus diffuser inlet flow angles were estimated by absolute velocity vector: radial component was calculated by means of diffuser mass flow rate and velocity module was calculated by flow condition at section 20 (temperature and dynamic pressure).

Section 20 was at the same radius for each configuration $\left(R_{20} / R_{2}=1.16\right)$. Thus, even if this section was not exactly located where the diffuser parallel wall started, comparison between different configurations was performed on the same radius.

The model was instrumented with several dynamic pressure sensors $\left(\mathrm{PCB}^{\circledR}\right.$ piezoelectric sensor with a natural frequency about $350 \mathrm{kHz}$ ) in order to study the inception, evolution, and characteristics of stall. The dynamic pressure sensors' (PS in the following exposition) position is defined in Table 2. Figure 4 shows their position for $\mathrm{DR}=1.3$ diffuser.

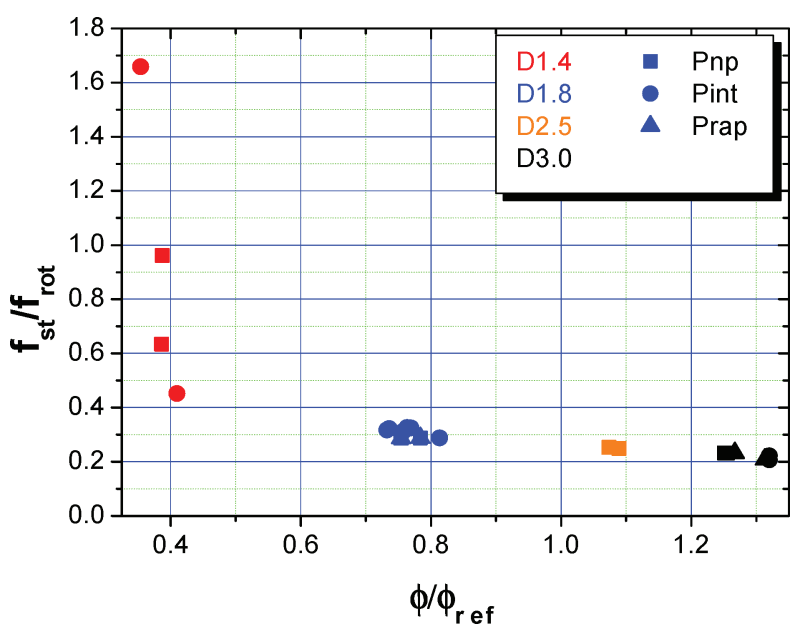

FIGURE 5

Stall inception frequencies (DR $=1.7)$. 
TABLE 2

Instrumentation Details

\begin{tabular}{cllccc}
\hline Section & \multicolumn{1}{c}{ Description } & \multicolumn{1}{c}{ TC } & \multicolumn{1}{c}{ TP } & SP & PS \\
\hline 200 & Model inlet & 2; spaced at $180^{\circ}$ & 4 ; spaced at $90^{\circ}$ & 4 ; spaced at $90^{\circ}$ & \\
00 & Stage inlet & 2; spaced at $180^{\circ}$ & 4 ; spaced at $90^{\circ}$ & 4 ; spaced at $90^{\circ}$ & \\
10 & Impeller inlet & & & & 2, spaced at $90^{\circ}$ \\
2 & Impeller outlet & 4; spaced at $90^{\circ}$ & & & \\
20 & Parallel wall diffuser inlet & 2; spaced at $180^{\circ}$ & 4 ; spaced at $90^{\circ}$ & 4 ; spaced at $90^{\circ}$ & 3, spaced at $45^{\circ}$ \\
$30^{(1)}$ & Diffuser mean radius & & & & 2, spaced at $45^{\circ}$ \\
40 & Outlet diffuser & 2; spaced at $180^{\circ}$ & 4 ; spaced at $90^{\circ}$ & 4 ; spaced at $90^{\circ}$ & 3, spaced at $45^{\circ}$ \\
60 & Volute outlet & 4; spaced at $90^{\circ}$ & 4 ; spaced at $90^{\circ}$ & 4 ; spaced at $90^{\circ}$ & \\
\hline
\end{tabular}

${ }^{(1)}$ only for DR $=1.3$.

\section{FREQUENCIES}

The analysis of dynamic pressure sensor signals was used to detect the inception of stall. When a sub-synchronous frequency appeared, the correspondent value of mass flow coefficient was recorded. Analysis of differences among signal of the sensors in different sections allows us to understand which is the critical component. A further help comes from the analysis of the ratio between the stall and rotation frequencies. For all the configurations tested, diffuser was the critical component, with a stall frequency about $20-40 \%$ of the rotation one. Generally, diffuser rotating stall is characterized by lower frequency ratio, but these values are typical for this kind of machine, as experiences done on field have shown. In Figure 5 the observed stall frequencies are reported for the configuration with $\mathrm{DR}=1.7$.

Essentially, with this configuration ( $\mathrm{DR}=1.7)$, stall always takes place in the diffuser. The only exception is the configuration with diffuser width of $1.4 \mathrm{~mm}$. That configuration leads to almost no-stalling diffuser, with surge occurring before. Sometimes an impeller stall is encountered (very high stall and rotation frequency ratio). When the intermediate pinch is used, a hyperrotating stall is present as first asynchrony frequency (Figure 6).

It is difficult to find an explanation for this phenomenon. The only reference the authors found in the literature about a hyperrotating stall is the one observed by Kobayashi et al. (1990) and

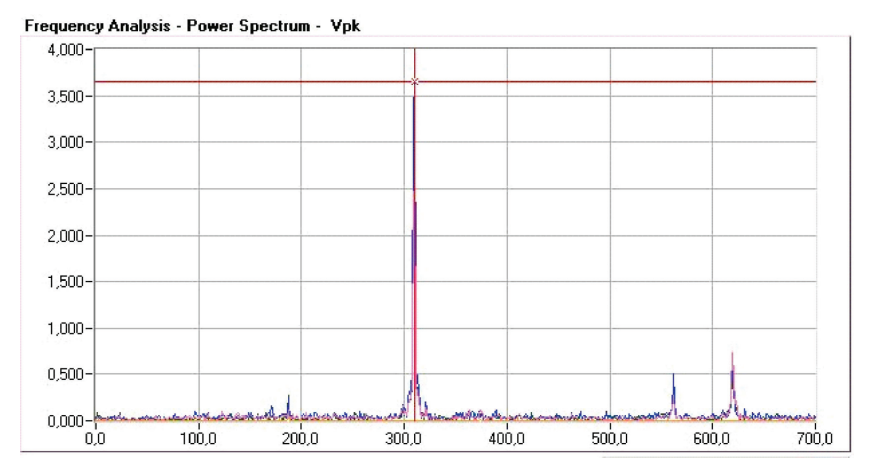

FIGURE 6

Starting stall spectrum $\left(\mathrm{f}_{\mathrm{st}} / \mathrm{f}_{\text {rot }}=1.68\right)(\mathrm{D}=1.4, \mathrm{DR}=1.7$, $\mathrm{P}=\mathrm{ra}, \mathrm{M}_{\mathrm{u}}=0.5$, sec. 20).
Madhavan and Wright (1985), the last reference in a completely different situation (blower, high flow rate).

When the configurations with $\mathrm{DR}=1.3$ were tested, things changed considerably in terms of machine working range: stall occurs for greater flow coefficient. Nevertheless, diffuser was always the critical component and the stall frequency range remained the same. No hyper-rotating stall was found (Figure 7).

\section{DIMENSION AND LOBE NUMBER}

A useful tool in understanding stall characteristics and evolution is the evaluation of the signal angular shift between sensors with a known angular distance at the same section (same radial position). The phase shift between sensors signal $(\triangle \varphi)$ divided by their geometrical angular distance $(\Delta \alpha)$ gives the number of lobes.

The sensor signal analysis itself gives information about the stall extension within the diffuser. In the configurations tested, the stall was generally composed of two lobes rigidly rotating at the frequencies reported above. The signals acquired generally

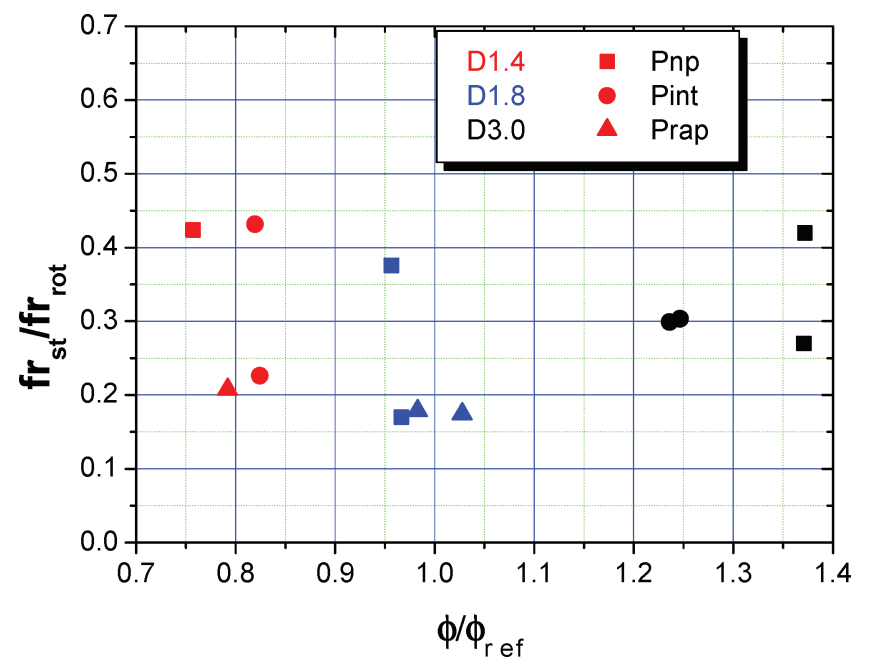

FIGURE 7

Stall inception frequencies $(\mathrm{DR}=1.3)$. 


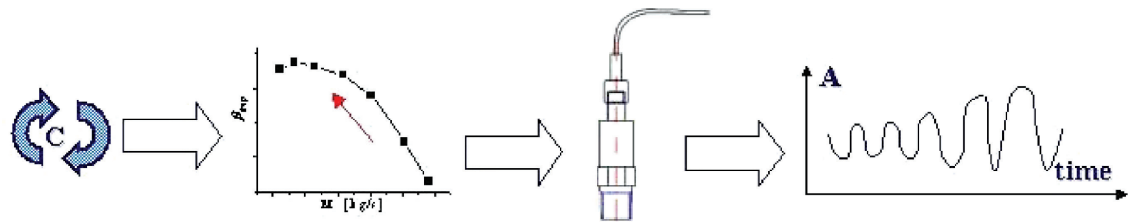

FIGURE 8

Sketch of streaming procedure.

had a quasi-sinusoidal trend with stall frequency, much bigger than the other component; all the diffuser is involved by stall.

This kind of analysis is good only if the stall has a behavior comparable with a solid body with negligible deformation during its motion. Phasing between the signals was very stable during the analysis. Then it is possible to affirm that few deformations of the stall zone are present.

\section{STREAMING-TIME ANALYSIS}

An interesting analysis on stall can be done analyzing the signal of dynamic pressure sensors when the circuit throttle valve is slowly closed with continuity from overflow to shut down (Figure 8). In this way it is possible to analyze the dynamic pressure transition due to stall inception and surge. During the throttle valve closing, data from dynamic pressure sensors was acquired and stored in a personal computer. A post-processor routine allows the analysis of that data. The post-processor analysis allows one to detect the instability inception. In this case, the comparison between sensors in different sections is particularly interesting. In Figures 9 and 10 examples of this analysis are reported. Abscissa is the time in which the single sample is acquired.

In Figure 9 an example of stall inception is reported. It is possible to notice how the stall high oscillation starts and develops in a few tenths of a second. It is also possible to notice that section 40 sensor signal is earlier than the section 30 and 20 ones. In Figure 10 an example of this analysis applied to starting surge is reported. Sensor of section 10 feels the typical surge pressure fluctuation earlier than the diffuser sensors.

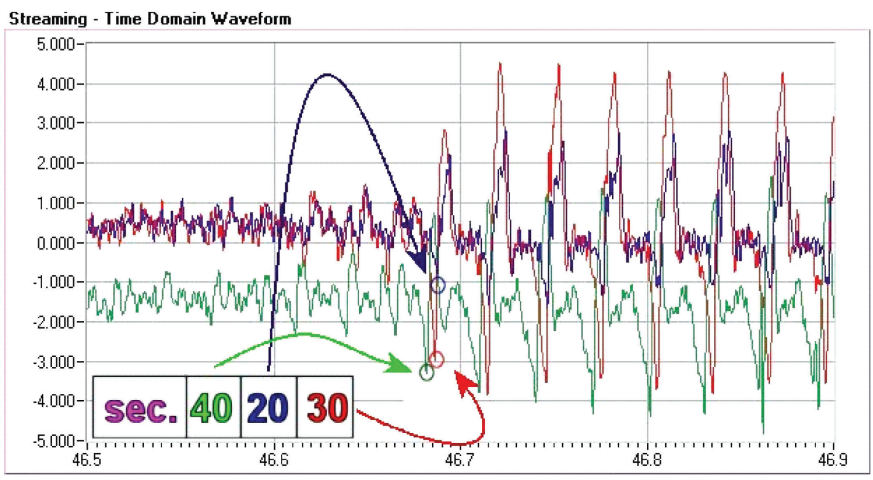

FIGURE 9

Stall inception $\left(\mathrm{D}=1.8, \mathrm{DR}=1.3, \mathrm{P}=\right.$ int, $\left.\mathrm{M}_{\mathrm{u}}=0.5\right)$.

\section{WATERFALL}

Starting from data acquired for the streaming analysis it is possible to apply another routine that, extracting the frequency components, allows the study of stall evolution during all the working range in frequency domain (Figure 11). An analysis window is superimposed to analyzed data. The frequency components of this portion of data are estimated using the power spectrum analysis. Then the analysis window is moved to the following portion of data keeping an overlap with the previous one. The same process is repeated until all the data is processed. All the power spectra are collected in a 3-D graph: frequency, time, and amplitude.

For the sake of simplicity, in the final graph the amplitude values are reported with a color scale. In Figure 12 a commented example is reported.

Frequencies are reported in the ordinate axis, whereas the acquisition number is reported in the abscissa axis (corresponding to different mass flow rate). Color intensity in each point represents the amplitude of that frequency component for that instant. A vertical section of that graph represents the signal spectrum analysis of one sensor for that time. Therefore it is possible to see the stall inception frequency and how it changes. Moreover, the first harmonics and the round signal are present. For low mass flow rate surge characteristic, low frequency pressure oscillations are also present. They were cut out from the graph since their amplitudes were so high that they would have scaled all the other graph frequencies. Surge is still locatable with the strong stall frequency perturbation. Generally, the previous analysis is done closing the throttle from maximum mass flow rate condition to deep surge and then opening. Sometimes the opening

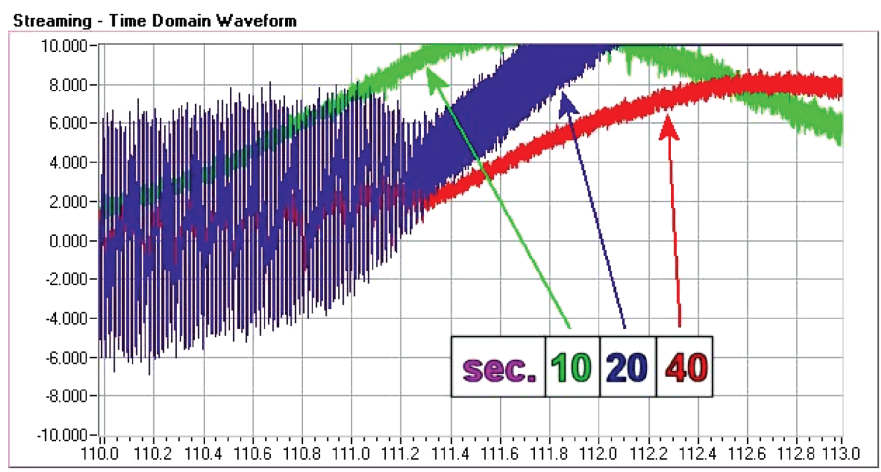

FIGURE 10

Surge inception $\left(\mathrm{D}=2.5, \mathrm{DR}=1.7, \mathrm{P}=\right.$ int, $\left.\mathrm{M}_{\mathrm{u}}=0.5\right)$. 


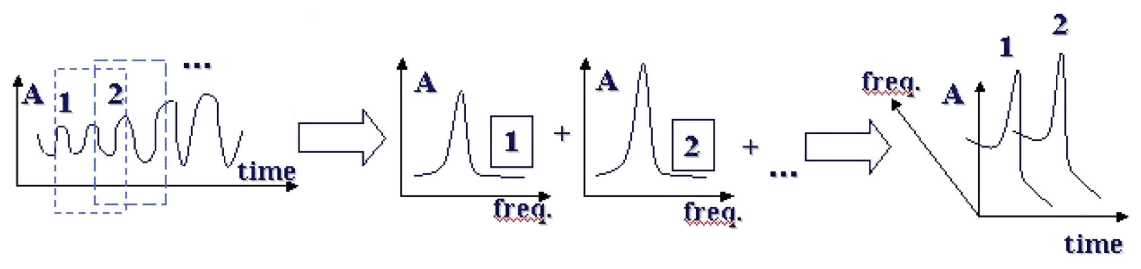

FIGURE 11

Sketch of waterfall procedure.

operation is faster. It is thus possible with this methodology to summarize diffuser behavior in terms of rotating stall, and surge in a single image. For this reason the waterfall analysis will be used to compare the stall behavior for the different configurations tested.

\section{TEST PARAMETER INFLUENCE ON STALL}

Due to the great number of geometries tested it is possible to analyze their influence on stall behavior. As previously said, the test parameters are:

1. Peripheral Mach Number $\left(\mathrm{M}_{\mathrm{u}}\right)$

2. Diffuser Width (D)

3. Diffusion Ratio (DR)

4. Pinch Shape (P)

Tests showed a very small influence on stall behavior of Mach number, pinch shape, and diffuser width. Of course all these parameters strongly influence the critical mass flow rate and the overall machine performance, but these aspects are out of the present articles aim (for details see Ferrara et al. (2002a), Ferrara et al. (2002b)). In Figures 13-16, some examples of the

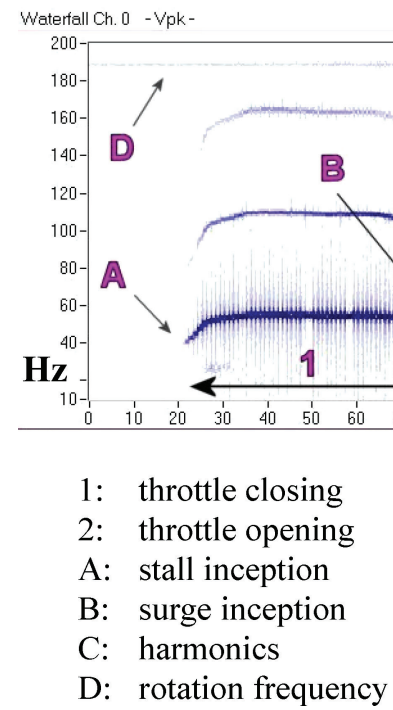

FIGURE 12

Waterfall analysis.

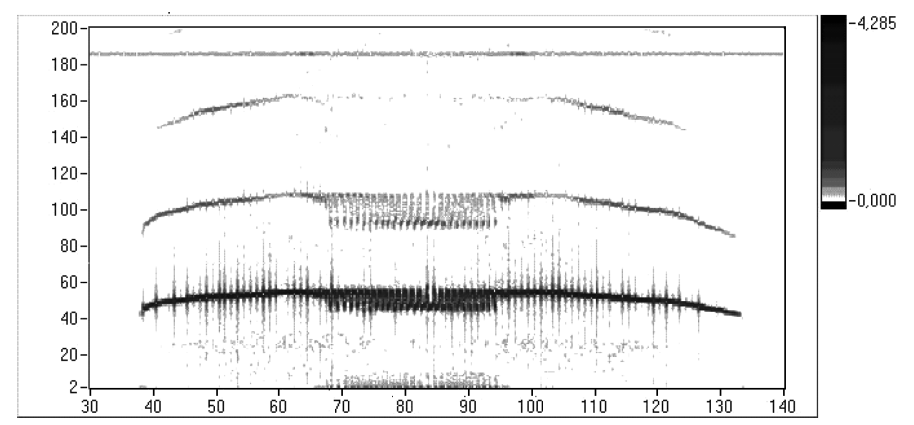

FIGURE 13

Waterfall analysis $\left(\mathrm{D}=3.0, \mathrm{DR}=1.7, \mathrm{P}=\mathrm{np}, \mathrm{M}_{\mathrm{u}}=0.5\right.$, sec. 20).

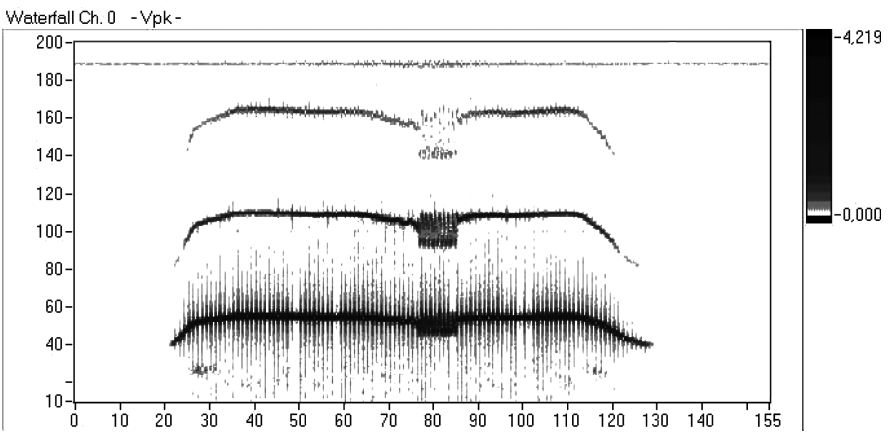

FIGURE 14

Waterfall analysis $\left(\mathrm{D}=3.0, \mathrm{DR}=1.7, \mathrm{P}=\mathrm{ra}, \mathrm{M}_{\mathrm{u}}=0.5\right.$, sec. 20).

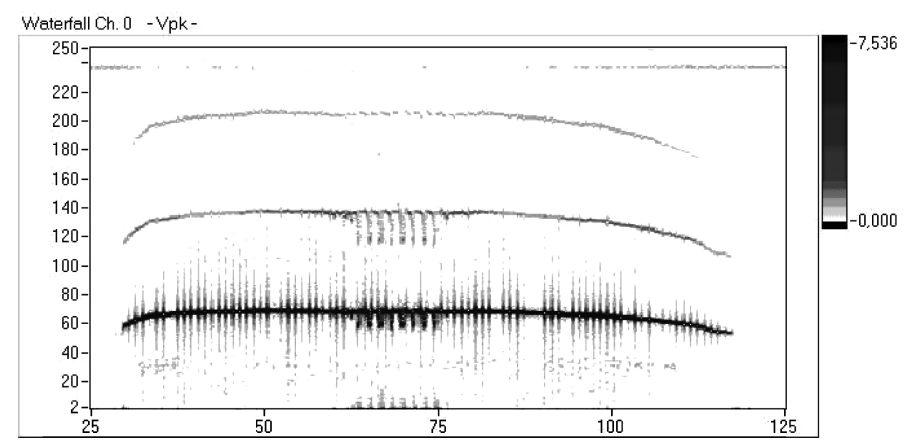

FIGURE 15

Waterfall analysis $\left(\mathrm{D}=3.0, \mathrm{DR}=1.7, \mathrm{P}=\mathrm{np}, \mathrm{M}_{\mathrm{u}}=0.65\right.$, sec. 20). 


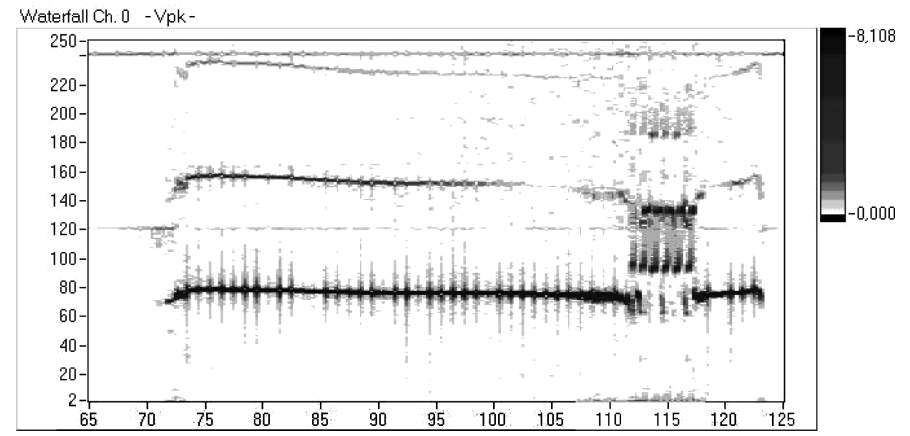

FIGURE 16

Waterfall analysis $\left(\mathrm{D}=1.8, \mathrm{DR}=1.7, \mathrm{P}=\right.$ int, $\mathrm{M}_{\mathrm{u}}=0.65$, sec. 20).

waterfall analysis for a base configuration and the configurations with different $\mathrm{D}, \mathrm{P}$, and $\mathrm{M}_{\mathrm{u}}$ are reported.

As can be clearly seen, the stall behavior is the same: it is characterized by a single frequency (and few harmonics) and it is stable until surge is reached. In particular, Mach number variation generally scales the stall frequencies leaving approximately unchanged the main structure of stall evolution and the ratio between stall and rotating frequency.

On the contrary, diffusion ratio seems to have great influence on stall behavior. In Figures 17 and 18, the waterfall analysis of the same configuration with different diffusion ratio is reported. This is an example, but the same thing happens for all the other configurations with the same diffusion ratio. As can be seen, a new stall structure appears during the evolution. In addition, it is possible to notice how sharp the passage is between the frequencies of the two phenomena. Three different stall structures following each other can be distinguished (A1, A2, and A3 in Figure 18).

To highlight the sharp change in stall frequency, in Figures 19 and 20 the streaming analyses of stall changing pattern are reported. See Figure 18 for references. For the flow coefficients between points A 2 and A3 indicated in Figure 18, the spectrum

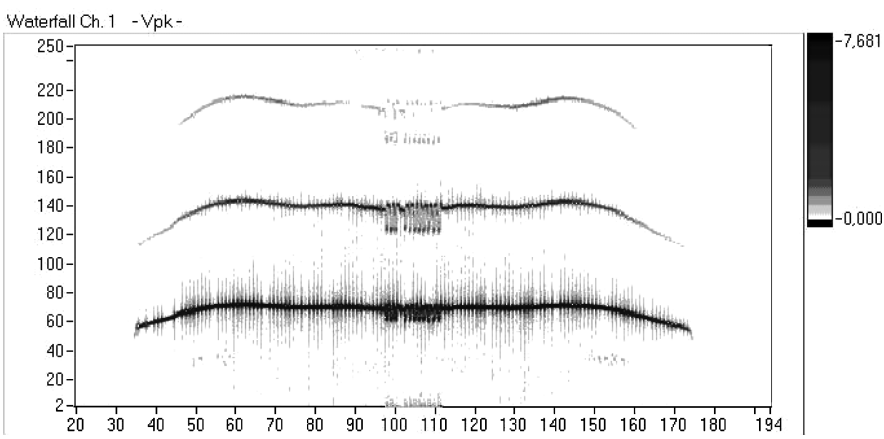

FIGURE 17

Waterfall analysis $\left(\mathrm{D}=3.0, \mathrm{DR}=1.7, \mathrm{P}=\right.$ int, $\mathrm{M}_{\mathrm{u}}=0.65$, sec. 20).

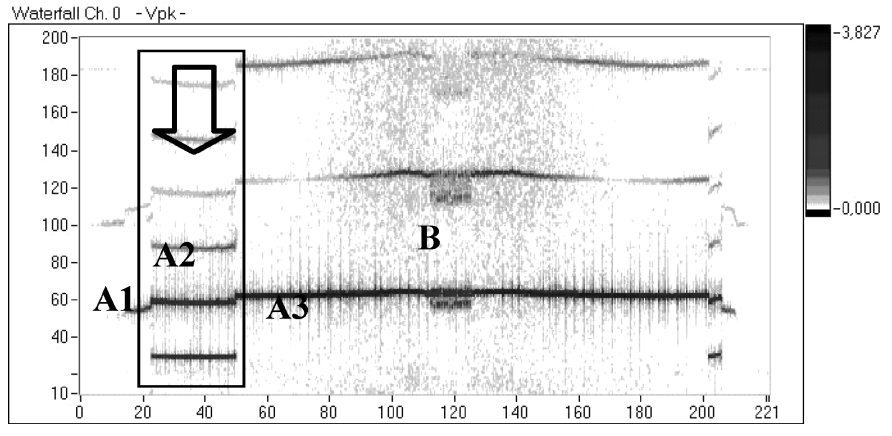

FIGURE 18

Waterfall analysis $\left(\mathrm{D}=3.0, \mathrm{DR}=1.3, \mathrm{P}=\right.$ int, $\mathrm{M}_{\mathrm{u}}=0.65$, sec. 20).

of the dynamic pressure measured has a shape similar to that shown in Figure 21.

The reason for this complex stall pattern with respect to the other one can most likely be associated with the different volute influence on diffuser flow. In the second configuration (smaller diffusion ratio) the volute is closer to the impeller exit and then the diffuser discharge conditions are quite different. Absolute tangential velocity modules becomes greater while the absolute velocity angle, measured with respect to the tangential direction, becomes smaller (due to the smaller friction losses).

The velocities at the diffusers exit for the shorter diffusers can be almost twice that of the longer diffuser while the angles are about $7-14^{\circ}$ smaller. This can make the flow condition critical at volute inlet. The volute design also enforces these possibilities: Firstly, it is a constant cross section volute, Secondly, the cross section was the same for the two configurations. It is thus possible that this stage part becomes the trigger for stall inception. The stability decrease of the stage characterized by this configuration is evident looking at the results obtained in terms of diffuser critical angles (for details see Ferrara et al. (2002a, 2002 b) - the shorter diffuser critical angles are bigger then for the longer one. It is also possible to notice that the stall evolution

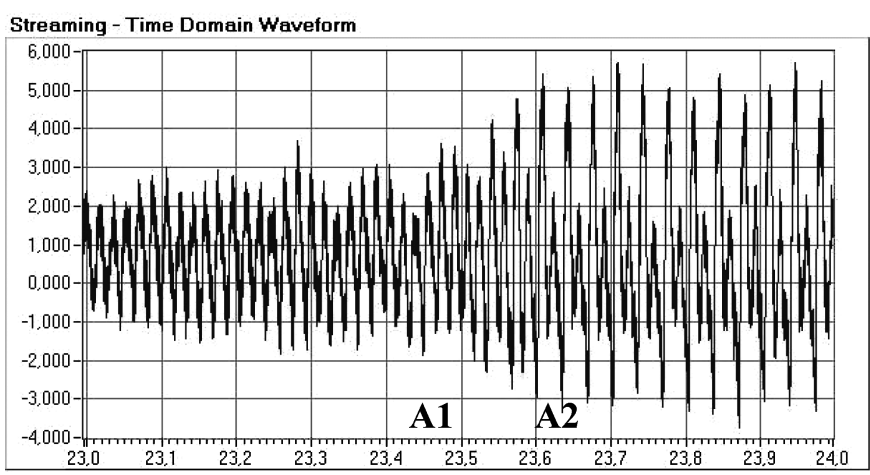

FIGURE 19

Stall change $\left(\mathrm{D}=3.0, \mathrm{DR}=1.3, \mathrm{P}=\right.$ int, $\mathrm{M}_{\mathrm{u}}=0.5$, sec. 20$)$. 


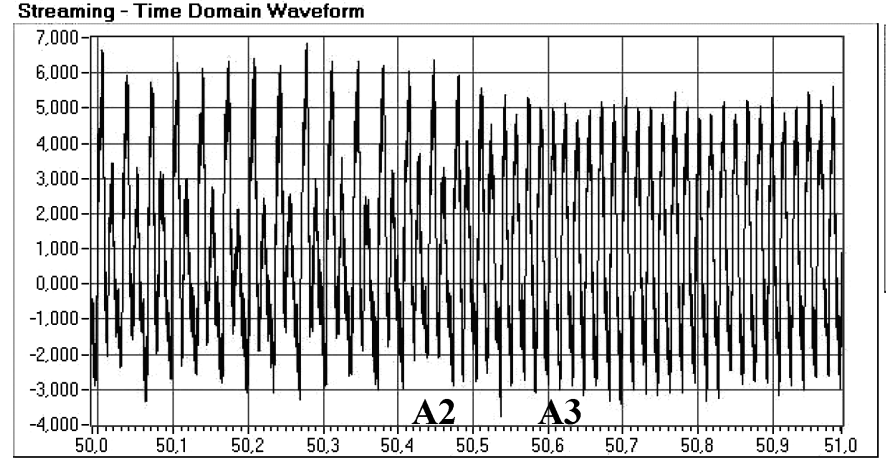

FIGURE 20

Stall change $\left(\mathrm{D}=3.0, \mathrm{DR}=1.3, \mathrm{P}=\right.$ int, $\mathrm{M}_{\mathrm{u}}=0.5$, sec. 20$)$.

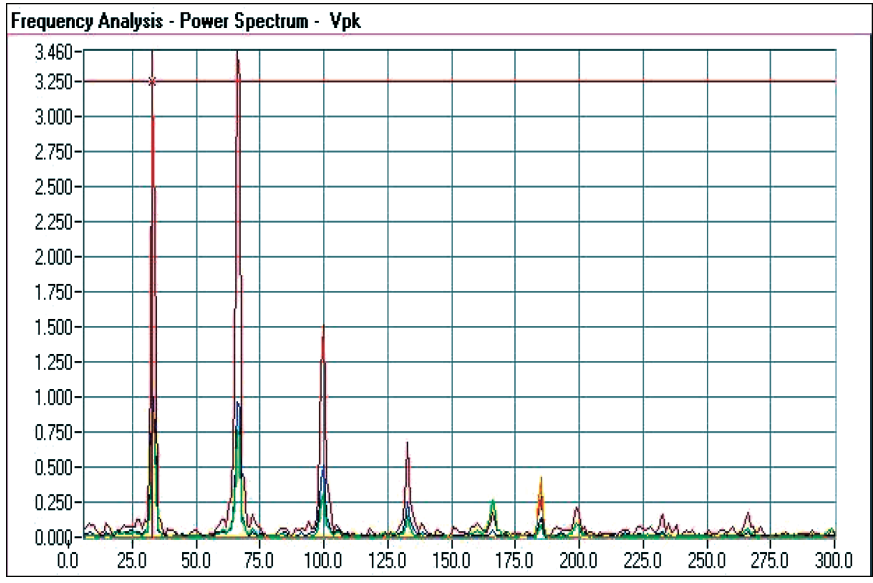

FIGURE 21

Sensor signal power spectrum $(\mathrm{D}=3.0, \mathrm{DR}=1.3, \mathrm{P}=\mathrm{int}$, $\mathrm{M}_{\mathrm{u}}=0.5$, sec. 20).

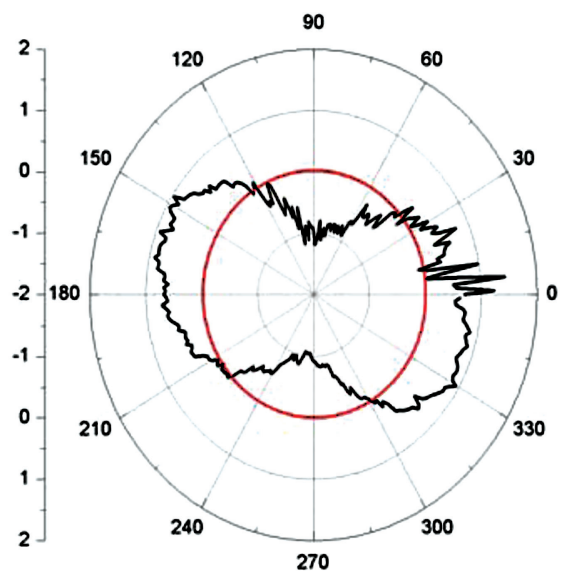

FIGURE 22

Stall pressure distribution at Section 20 for pattern A1.

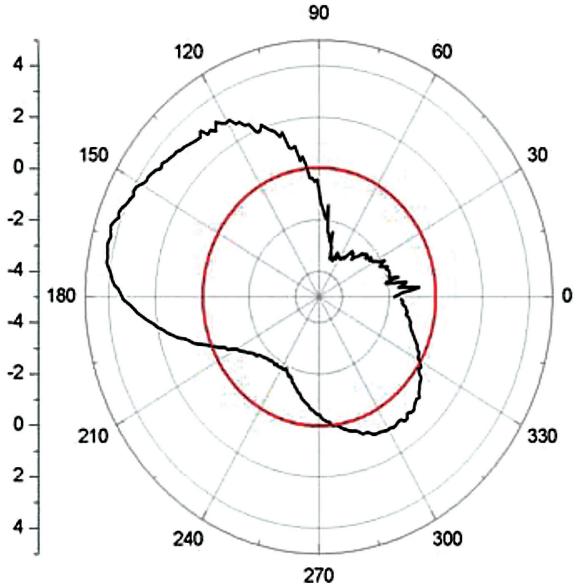

FIGURE 23

Stall pressure distribution at Section 20 for pattern A2.

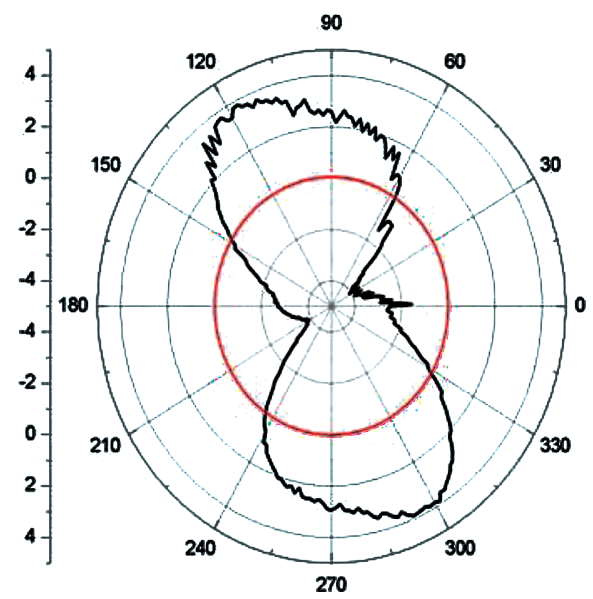

FIGURE 24

Stall pressure distribution at Section 20 for pattern A3.

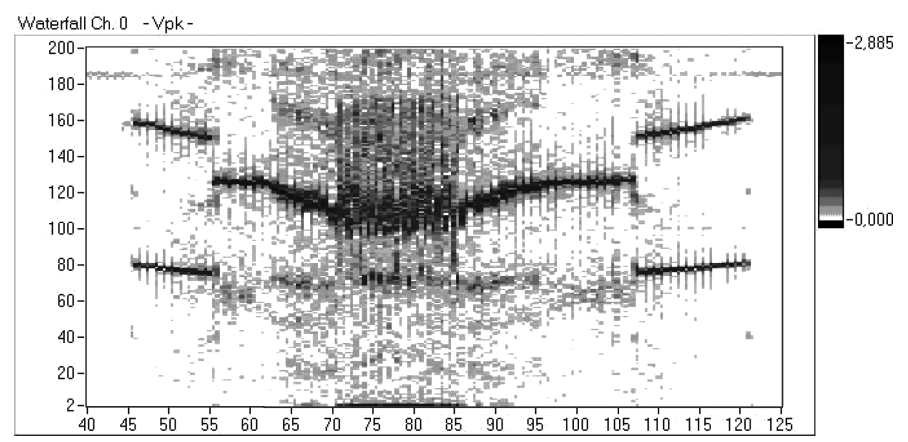

FIGURE 25

Waterfall analysis $\left(\mathrm{D}=1.4, \mathrm{DR}=1.3, \mathrm{P}=\right.$ int, $\mathrm{M}_{\mathrm{u}}=0.5$, sec. 20). 
in the two cases is quite similar, except for the zone where the comb pattern is present.

Nevertheless, since the complex stall structure is present only for a portion of the machine working range, the condition that generates it shall be limited. It is not easy to find an explanation for this phenomenon: it could depend on volute influence that, in this configuration, is closer to the impeller.

Considering the hypothesis, widely verified in reported tests, of stall moving like a rigid body it is possible to show the diffuser pressure distribution (AC value), starting from the pressure history measured by a dynamic pressure sensor. Results are reported in Figures 22-24 for Section 20, taking into account that stall was characterized by two lobes. Refer to Figures 19 and 20 for the stall structure.

It is interesting to note that for pattern A2 the pressure distribution is strongly asymmetric (Figure 23).

As for $\mathrm{DR}=1.7$ as well as $\mathrm{DR}=1.3$, the diffuser $1.4 \mathrm{~mm}$ width shows a particular behavior (see Figure 25). It is possible to notice a single diffuser stall pattern that becomes impeller stall for low flow coefficient values. It is sufficient to have a diffuser $1.5 \mathrm{~mm}$ width (Figure 26), to return to the previously described pattern.

\section{VISUALIZATION}

Even if some theoretical explanation of stall development in the diffuser can be done, it is still hard to understand what is really happening. For this reason the previously seen disposition was chosen for the dynamic pressure sensors of the short vaneless diffuser dynamic sensor. Interpolating the values read by each sensor it is possible to obtain an instantaneous pressure map. Repeating the same process for successive instants it is possible to visualize diffuser pressure map evolution as well as stall behavior. This kind of analysis is still in progress; in particular, it is necessary to have a very good sensor calibration to compare the pressure distribution revealed by each sensor. In Figure 27 an example of instant pressure interpolation is reported.

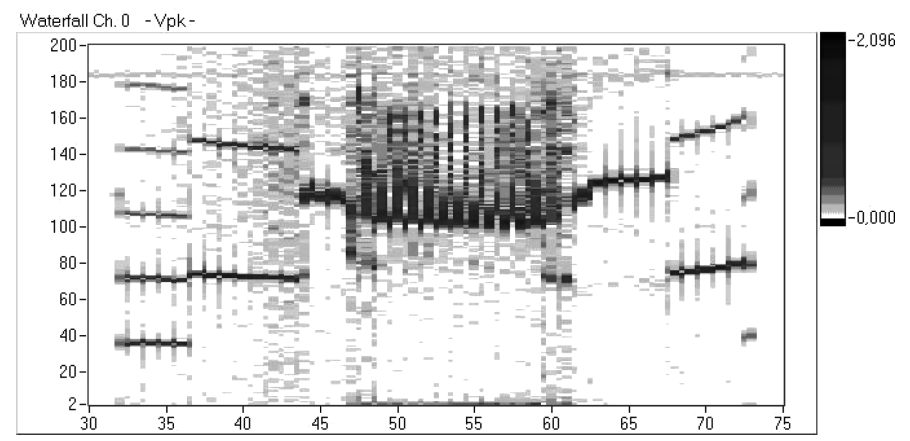

FIGURE 26

Waterfall analysis $\left(\mathrm{D}=1.5, \mathrm{DR}=1.3, \mathrm{P}=\mathrm{ra}, \mathrm{M}_{\mathrm{u}}=0.5\right.$, sec. 20).

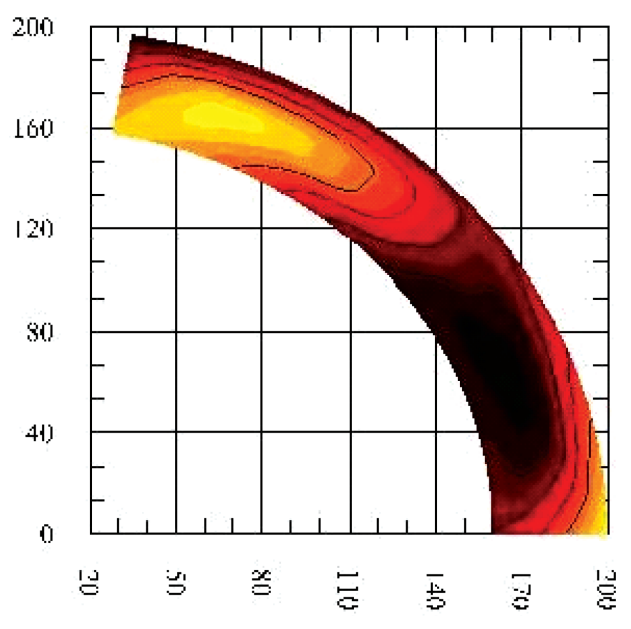

FIGURE 27

Pressure distribution $\left(\mathrm{D}=3.0, \mathrm{DR}=1.3, \mathrm{P}=\right.$ int, $\left.\mathrm{M}_{\mathrm{u}}=0.65\right)$.

This kind of analysis, if properly done, enables the whole stall to be characterized. Determining the lobe number, their disposition and dimension would be immediate.

\section{CONCLUSIONS}

A wide-ranging study on the influence of diffuser geometry on high pressure last stage centrifugal compressors was planned by General Electric-Nuovo Pignone and Department of Energy Engineering "S. Stecco." The main purpose of this study was to characterize the geometry modifications in terms of stall behavior and stage performance.

The use of dynamic pressure sensors, their position, and the applied analysis methodology, provided much information on stall inception and evolution. A wide-ranging analysis on both in time and in frequency domains was carried out and the results obtained reported in this article. They can be summarized as follows:

1. Pinch geometry seems not to modify stall evolution.

2. Diffuser width seems not to modify stall evolution, excluding the case of $\mathrm{D}=1.4 \mathrm{~mm}$ that leads to an almost no stalling diffuser.

3. Mach number variation usually only scales the stall frequencies leaving approximately unchanged the main structure of stall evolution and the ratio between stall and rotating frequency. Only for short diffusers was some influence on stall pattern observed.

4. The changing on diffusion ratio shows big differences both on the stall pattern and on the stall inception point. The more complex stall pattern and the smaller stall inception flow coefficient of the shorter diffuser seem due to volute influence.

5. For all the configurations tested, the stall seems to be bilobed and the entire diffuser seems involved. This will be better seen when the stall view analysis is adjusted. 
It is important to notice that even if tested geometries seem not to influence stall evolution, they do strongly influence stall inception flow coefficient and stage performance.

\section{NOMENCLATURE}

$\begin{array}{ll}\triangle \alpha & \text { eometrical angular distance } \\ \triangle \varphi & \text { hase shift } \\ \phi_{\text {ext }} & \text { impeller diameter } \\ \phi_{\text {ref }} & \text { reference flow coefficient } \\ \mathrm{a}_{00} & \text { sound speed at sec. } 00 \\ \mathrm{~b}_{20}, \mathrm{D} & \text { diffuser width } \\ \mathrm{DR}=\mathrm{r}_{5} / \mathrm{r}_{2} & \text { diffusion ratio } \\ \mathrm{f}_{\mathrm{st}} & \text { stall frequency } \\ \mathrm{f}_{\mathrm{rot}} & \text { rotation frequency } \\ \mathrm{P} & \text { pinch shape } \\ \mathrm{M}_{\mathrm{u}}=\mathrm{u}_{2} / \mathrm{a}_{00} & \text { peripheral Mach number } \\ \mathrm{r}_{2} & \text { outlet impeller radius } \\ \mathrm{r}_{5} & \text { outlet diffuser radius } \\ \mathrm{SP} & \text { static pressure tap } \\ \mathrm{TC} & \text { thermocouple } \\ \mathrm{TP} & \text { total pressure Kiel probe } \\ \mathrm{u}_{2} & \text { peripheral velocity at impeller outlet }\end{array}$

\section{REFERENCES}

Abdelhamid, A. N. 1983. Effects of vaneless diffuser geometry on flow instability in centrifugal compression systems. ASME Paper No. 81-GT-10; Canadian Aeronautics and Space Journal 29(3):259288.

Camp, T. R., and Day, I. J. 1998. A study of spike and modalstall phenomena in a low speed axial compressor. Journal of Turbomachines.

Ferrara, G., Ferrari, L., Mengoni, C. P., De Lucia, M., and Baldassarre, L. 2002. Experimental investigation and characterization of the rotating stall in a high pressure centrifugal compressor: Part I: Influ- ence of diffuser geometry on stall inception, ASME Paper GT-200230389.

Ferrara, G., Ferrari, L., Mengoni, C. P., De Lucia, M., and Baldassarre, L. 2002. Experimental investigation and characterization of the rotating stall in a high pressure centrifugal compressor Part II: Influence of diffuser geometry on stage performance, ASME Paper GT-200230390 .

ISO 5167. 1980. Measurement of fluid flows by means oforifice plates, nozzles and venturi tubes inserted incircular cross-section conduits running full.

Jansen, W. 1964. Rotating stall in a radial vaneless diffuser. ASME Paper No. 64-FE-6; Transactions of the ASME Journal of Basic Engineering 750-758.

Kobayashi, H., Nishida H., Takagi, T., and Fukoshima, Y. 1990. A study on the rotating stall of centrifugal compressors (2nd Report, Effect of vaneless diffuser inlet shape on rotating stall). Transactions of the Japan Society of Mechanical Engineers (B Edition) 56(529):98103.

Madhavan, S., and Wright, T. 1985. Rotating stall caused by pressure surface flow separation on centrifugal fan blades. Transactions of the ASME Journal of Engineering for Gas Turbomachinery and Power 107:775-781.

Nishida, H., Kobayashi, H., Takagi, T., and Fukoshima, Y. 1988. A study on the rotating stall of centrifugal compressors (1st Report, Effect of vaneless diffuser width on rotating stall). Transactions of the Japan Society of Mechanical Engineers (B Edition) 54(499):589594.

Senoo, Y., and Kinoshita, Y. 1977. Influence of inlet flow conditions and geometries of centrifugal vaneless diffuser on critical flow angle for reverse flow. Transactions of the ASME Journal of Fluids Engineering 98-103.

Senoo, Y., and Kinoshita, Y. 1978. Limits in rotating stall and stall in vaneless diffuser of centrifugal compressors. ASME Paper No. 78-GT-19.

Senoo, Y., Kinoshita, Y., and Ishida, M. 1977. Asymmetric flow in vaneless diffuser of centrifugal blowers. Transactions of the ASME Journal of Fluids Engineering 99(1):104-114. 

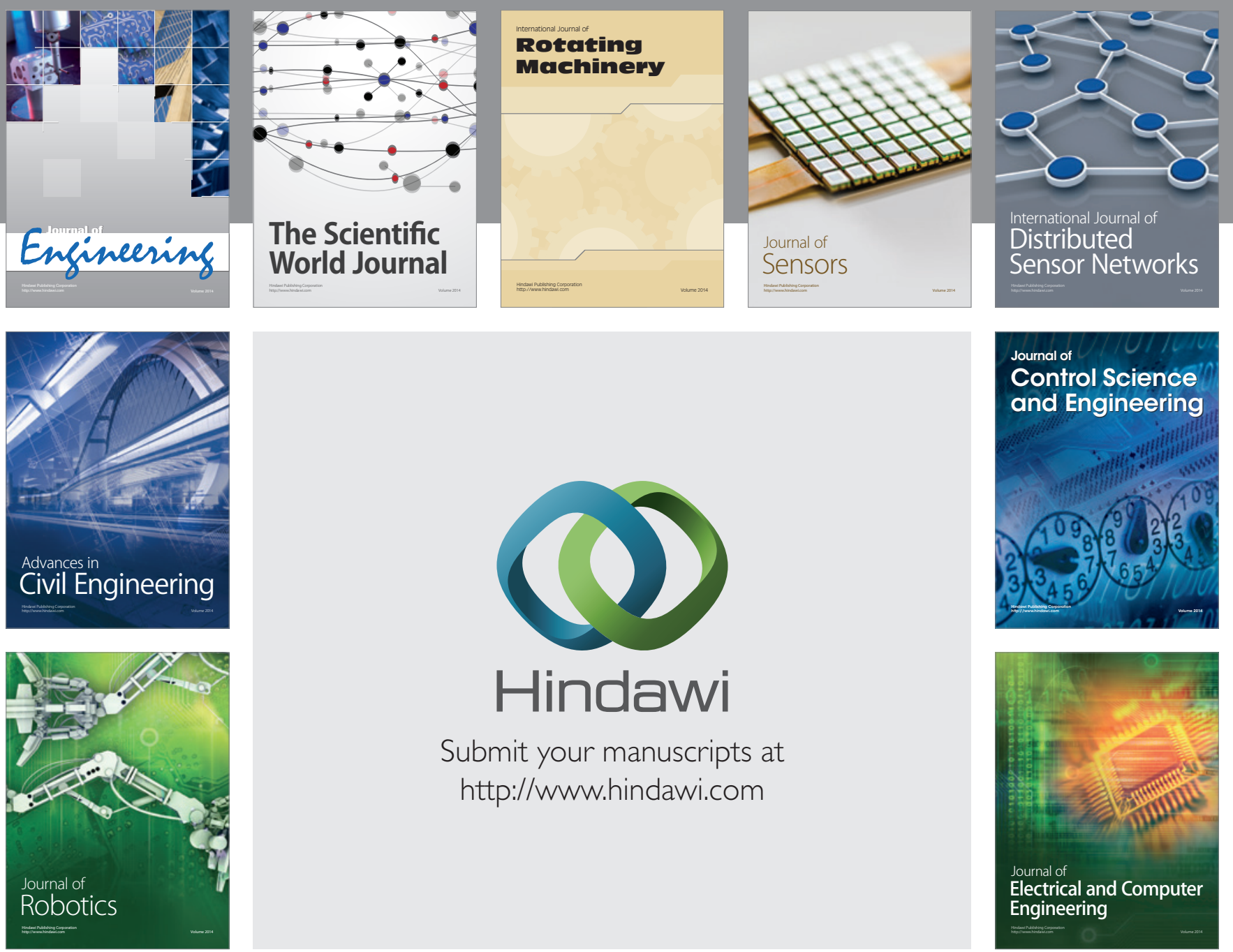

Submit your manuscripts at

http://www.hindawi.com
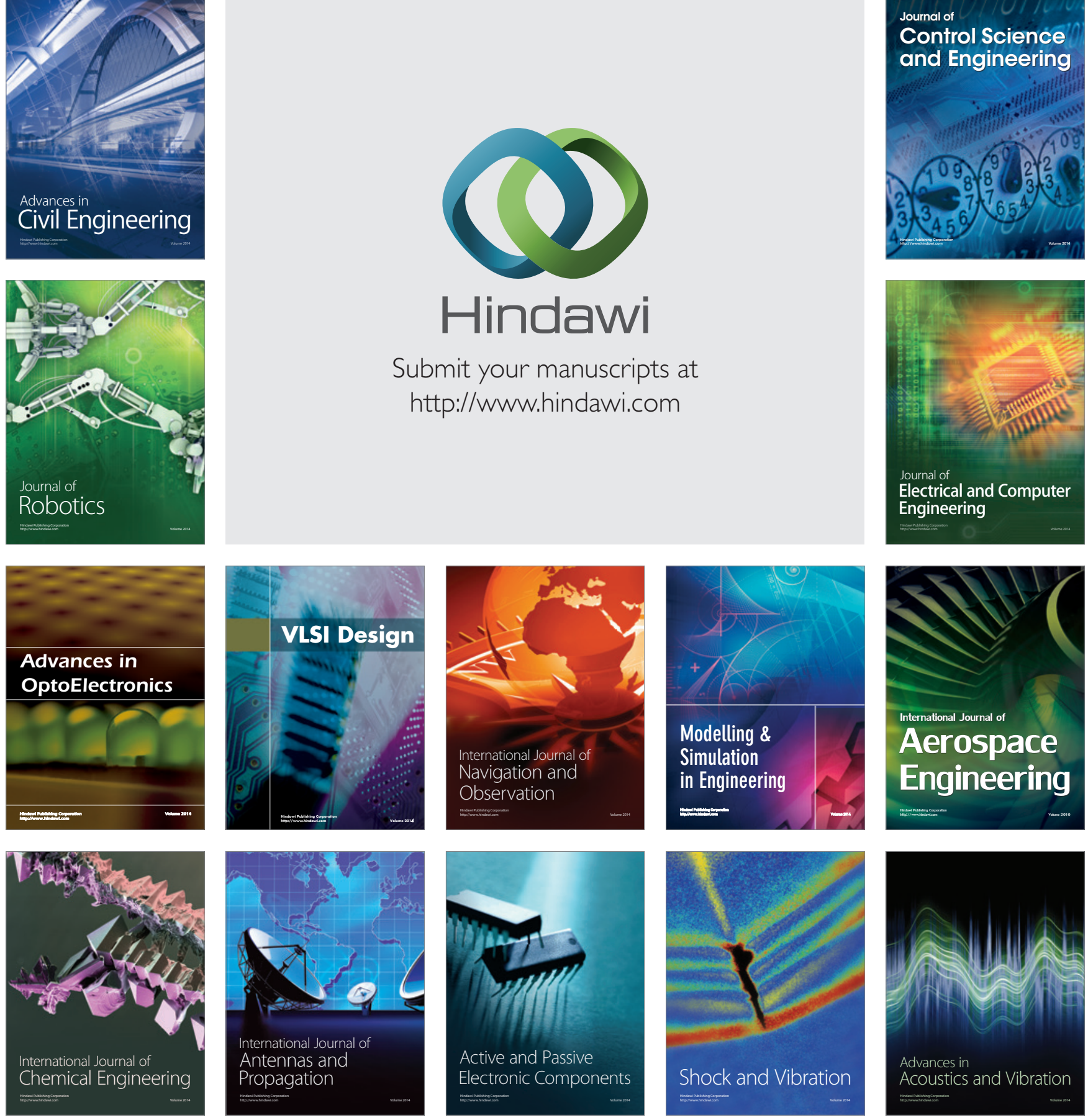